\title{
Social and environmental conditions related to Mycobacterium leprae infection in children and adolescents from three leprosy endemic regions of Colombia
}

\author{
Héctor Serrano-Coll ${ }^{1}$, Hugo Rene Mora², Juan Camilo Beltrán², Malcolm S. Duthie ${ }^{3}$ and Nora Cardona-Castro ${ }^{4^{*}}$ (1)
}

\begin{abstract}
Background: Leprosy is is still considered a public health issue and in Colombia 7-10\% of new cases are found in children, indicating both active transmission and social inequality. We hypothesized that circulating antibodies against Natural Octyl Disaccharide-Leprosy IDRI Diagnostic (NDO-LID) (a combination of Mycobacterium leprae antigens) could reveal the social and environmental aspects associated with higher frequencies of $M$. leprae infection among children and adolescents in Colombia.

Methods: An observational cross-sectional study was conducted involving sampling from 82 children and adolescents (younger than 18 years of age) who had household contact with index leprosy patients diagnosed in the last 5 years. Data were analyzed through bivariate analysis made by applying a Pearson $x^{2}$ test for qualitative variables, while quantitative variables, depending on their distribution, were analyzed using either a Student's t-test or MannWhitney $U$ test. Multivariate analysis was performed using a multiple regression and binomial logistic approach.

Results: A bivariate analysis demonstrated that antibody titers against NDO-LID were significantly greater in children and adolescents with a low socioeconomic status that had: lived in vulnerable areas of the UAChR shared region; eaten armadillo meat; exposure of over 10 years to an index case and; not received BCG immunization. Moreover, a multivariate analysis showed that residing in the UAChR region has a strong association with a greater possibility of $M$. leprae infection.

Conclusions: $M$. leprae transmission persists among young Colombians, and this is associated with social and environmental conditions. An intensification of efforts to identify new leprosy cases in vulnerable and forgotten populations where $M$. leprae transmission continues therefore appears necessary.
\end{abstract}

Keywords: Leprosy, Antibodies, Children, Adolescents, Mycobacterium leprae

\section{Background}

Leprosy is a chronic infectious disease caused by Mycobacterium leprae, an alcohol-acid resistant bacillus that has a notable affinity for the skin and peripheral nerves [1]. From an epidemiological perspective, approximately 225,000 new leprosy cases are diagnosed each year around the world, with $8.9 \%$ of these cases occurring in children and adolescents [2, 3]. Furthermore, at the time

\footnotetext{
* Correspondence: ncardona@ces.edu.co

${ }^{4}$ Instituto Colombiano de Medicina Tropical, Faculty of Medicine, Escuela de Graduados - Universidad CES, Cra 43 A \# 52 Sur 99., Sabaneta, Colombia Full list of author information is available at the end of the article
}

of diagnosis $11 \%$ of these children already present with grade 2 disability increasing to $27.3 \%$ during their follow-up [3].

In Colombia, 300-500 new cases of leprosy are reported per year. Seven percent of the new leprosy cases occurred in children younger than 15 years of age, 59\% of these cases were in children 10-14 years of age $[4,5]$. Although there is a low prevalence of leprosy in Colombia as a whole $(<1 / 10000$ inhabitants), we have detected high transmission of $M$. leprae with certain at risk populations [5]. Thorough evaluation by physical examination, detection of antibodies against the gold

(c) The Author(s). 2019 Open Access This article is distributed under the terms of the Creative Commons Attribution 4.0 International License (http://creativecommons.org/licenses/by/4.0/), which permits unrestricted use, distribution, and reproduction in any medium, provided you give appropriate credit to the original author(s) and the source, provide a link to the Creative Commons license, and indicate if changes were made. The Creative Commons Public Domain Dedication waiver (http://creativecommons.org/publicdomain/zero/1.0/) applies to the data made available in this article, unless otherwise stated. 
standard antigen phenolic glycolipid (PGL)-I, and PCR detection of $M$. leprae DNA in nasal swabs and suspicious skin lesions have contributed to the detection of both new patients and infected but healthy household contacts (HHC). In addition, molecular epidemiology studies in Colombia have demonstrated intra-familiar $M$. leprae transmission [6]. Determining who is infected with $M$. leprae is complicated by the inability to isolate and culture the bacteria ex vivo. Antibodies against $M$. leprae are therefore considered as a simple proxy indicator of infection or, at a minimum, exposure. We previously reported that the synthetic mimetic of PGL-I, natural octyl disaccharide (NDO), can be combined with recombinant protein Leprosy IDRI Diagnostic (LID)-1 to provide the NDO-LID conjugate that can detect antibodies in the serum of leprosy patients and many HHC [7]. In patients, the magnitude of the antibody response correlates strongly with the level of infection [7].

Leprosy among children and adolescents has been correlated with certain social and environmental aspects, such as cohabiting with an index case, malnutrition, living in overcrowded households, and absence of bacillus Calmette-Guerin (BCG) immunization scars [4, 8]. Likewise, $M$. leprae infection could also be linked to interactions with potential animal reservoirs such as armadillos [9]. Importantly, as it was mentioned, $7 \%$ of Colombian cases occur in children younger than 15 years of age with approximately 3 of every 5 of these cases are in children aged 10-14 years old [4,5]. These data are alarming because they reveal ongoing and active transmission of $M$. leprae that, on top of a diagnostic lag, indicate shortcomings in the elimination efforts of leprosy control programs.

Given that detection of serum antibodies against $M$. leprae is more practical among at risk populations than direct detection methods (PCR and histopathology) [6], we hypothesized that these responses used in the context of various socioeconomic indicators could enhance our understanding of factors that contribute to the risk of $M$. leprae infection and development of leprosy. The objective of this study was therefore to determine the relationship between anti-NDO-LID antibodies (IgM, IgG, protein A titers (i.e. antibodies for both IgM and IgG)), taking into account that the levels of these immunoglobulins are strongly linked with $M$. leprae infection levels $[7,10]$, and social and environmental aspects that could be associated with a higher $M$. leprae infection rate in children and adolescents in the higher risk regions of Colombia.

\section{Methods}

\section{Study and sample description}

An observational cross-sectional study was conducted during 2015 and 2016 with a sample frame made of 82 selected children and adolescents 18 years and younger. Each had HHC with a leprosy patient that had been diagnosed in the last 5 years, and all were included for convenience sampling. The study population was derived from three geographical regions where the reporting of new leprosy cases is greater than other parts of Colombia: Uraba-Antioquia-Choco (UAChR; $n=18$ children), from the Caribbean Region (CR; $n=43)$, and from the Andean Region (AR; $n=21$ ).

\section{Sociodemographic and environmental characteristics compilation}

Sociodemographic characteristics were gathered using a $\mathrm{HHC}$ evaluation form implemented by the leprosy research team of the Instituto Colombiano de Medicina Tropical (ICMT). For the purposes of this study we focussed on: age, sex, socioeconomic status, geographic location, recorded armadillo consumption, BCG immunization status, and clinical form of the index case. The socioeconomic categories established by the Colombian government are named status 1. Low-low, 2.Low, 3. Medium-low, 4. Medium, 5. Medium-high, 6. High. Status 1, 2 and 3 corresponding to low status that included the people of scarce resources [11].

\section{Measurement of antigen-specific serum antibodies}

Serum samples were collected for subsequent serologic evaluation. Briefly each well of 96-well ELISA plates (Nunc-Immuno 96-well, Polysorp plates) was coated with $1 \mu \mathrm{g} / \mathrm{ml}$ NDO-LID antigen at room temperature then blocked using $100 \mu \mathrm{l}$ blocking buffer (1\% bovine serum albumin, BSA/ phosphate-buffered saline, PBS/ PBS + Tween20, PBS-T). Plates were incubated for $1 \mathrm{~h}$ with agitation at room temperature. Plates were washed (5 PBS-T $+2 \mathrm{PBS}$ ), and $50 \mu \mathrm{l}$ serum (1:200 dilution in BSA $0.1 \% /$ PBS/ PBS-T) was added, followed by a $1 \mathrm{~h}$ of incubation with agitation at room temperature. Subsequently, $50 \mu \mathrm{l}$ horseradish peroxidase (HRP)-conjugated detector diluted in BSA 0.1\%/ PBS/ PBS-T was added and plates incubated for $1 \mathrm{~h}$ with agitation at room temperature. Three detectors were evaluated: antihuman IgG, anti-human IgM and protein A (Rockland Immunochemicals Inc., Limerick, PA, USA). After incubation and washing, $50 \mu \mathrm{l}$ TMB substrate was added for $15 \mathrm{~min}$ in the dark at room temperature, then stopped by adding $25 \mu \mathrm{l} 1 \mathrm{~N}$ sulfuric acid. Optical densities (OD) were measured at $450 \mathrm{~nm}$ using an ELISA plate reader (Spectrophotometer Bio-Rad Xmark) [10]. Cut-off values were assessed as the average OD plus two standard deviations obtained from sera $(n=100)$ of healthy individuals that resided in an area not endemic for leprosy. Cut-off values of $0.127,0.226$ and 0.183 were obtained for Protein A, IgM and IgG, respectively. 


\section{Statistical analyses}

Data was analyzed using Excel and SPSS 24.0 software. Univariate analysis of qualitative variables was made via absolute and relative frequencies calculation. For quantitative variables summary measures like central tendency were performed. The distribution of variables was obtained using a Kolmogorov-Smirnov test. Bivariate analysis between qualitative variables was made by applying a Pearson $x^{2}$ test and quantitative variables were analyzed using a Student's t-test or Mann-Whitney U test, taking into account the distribution of these variables. Multivariate analysis was conducted using a multiple regression and binomial logistic approach. Significance level of $p$-value $<0.05$ were established for all analysis and a risk approximation was made using an odds ratio calculation (OR) with its own confidence interval $(95 \% \mathrm{CI})$.

\section{Ethics declaration}

This study was considered of minimal risk and was approved by the Instituto Colombiano de Medicina Tropical - Universidad CES ethics committee. After the aims of the study were explained, an informed consent form was signed by the guardians of participating children.

\section{Results}

\section{Sample characterization}

A total of 82 children and adolescents were included, of which $36(44 \%)$ were male and $46(56 \%)$ were female. The age range was 1 to 18 years of age, with a mean of 10.7 years (Table 1 ). Of note, the majority of these children and adolescents lived with an index leprosy case: 57 with a lepromatous case; 12 with a pure neural leprosy case, 10 with a dimorphic leprosy case, 2 with an intermediate leprosy case and 1 with a tuberculoid case.

\section{Relationship between antibody responses and socioeconomic status}

In accordance with the socioeconomic categories established by the Colombian government, we found that 42 (51\%) of the children and adolescents could be

Table 1 Sample characteristics of the study cohort

\begin{tabular}{llll}
\hline Index case of leprosy & $\begin{array}{l}\text { Sample size } \\
\mathrm{N}(\%) \mathrm{HHC}\end{array}$ & Sex, M/F N (\%) & $\begin{array}{l}\text { Average age } \\
\text { (range), years }\end{array}$ \\
\hline $\mathrm{I}$ & $2(2 \%)$ & $2(100 \% \mathrm{M})$ & $9(4-14)$ \\
$\mathrm{TT}$ & $1(1 \%)$ & $1(100 \% \mathrm{~F})$ & 11 \\
$\mathrm{D}$ & $10(12 \%)$ & $6 / 4(60-40 \%)$ & $12.4(5-17)$ \\
$\mathrm{LL}$ & $57(70 \%)$ & $23 / 34(40-60 \%)$ & $10.1(1-18)$ \\
$\mathrm{NL}$ & $12(15 \%)$ & $8 / 4(67-34 \%)$ & $13.1(7-18)$ \\
Total & 82 & $36 / 46(44 / 56 \%)$ & $10.7(1-18)$ \\
\hline
\end{tabular}

$M$ male, $F$ female, $H H C$ household contact, I indeterminate leprosy, $\pi$ tuberculoid leprosy, D dimorphic leprosy, LL lepromatous leprosy, NL neural leprosy, $N$ number considered of low-low socioeconomic status while 40 (49\%) could be considered of low or a medium-low status. When seropositivity against NDO-LID was compared in these two sample groups, the frequency of antiNDO-LID antibodies detected by Protein A (i.e. of both IgG and IgM isotypes) and IgM was highest among children of lower socioeconomic backgrounds (Table 2; $p$-value $=0.001$ ).

In addition to the simple presence of antibodies, we determined anti-NDO-LID antibody levels. Significantly stronger anti-NDO-LID Protein A $(p=0.001), \operatorname{IgM}(p=$ $0.011)$ and IgG $(p=0.032)$ responses were detected in children and adolescents of low-low status relative to those observed in samples from subjects of low or medium-low status (Fig. 1a). These data imply that, in addition to a greater rate of $M$. leprae infection, the overall levels of infection in each child or adolescent of low-low socioeconomic status are higher.

\section{Relationship between antibody responses and geographic region}

When assessing anti-NDO-LID antibodies detected by protein A and IgM according to the geographic origin of subjects, we found higher frequency of seropositivity in children and adolescents from UAChR $(P=0.0001$, see Table 2).

Antibody titers were also compared based on the geographic origin of the subject. We found that anti-NDOLID responses detected by Protein A, IgM and IgG in children and adolescents from UAChR region were significantly greater (all $p$-values $=0.0001$ ) than the levels measured among samples from the Caribbean and Andean regions (Fig. 1b). Taken together, these results suggest that children and adolescents from UAChR are under higher pressure for $M$. leprae infection than those that reside around the Caribbean or in the Andes.

\section{Antibody responses among children and adolescents of low-low socioeconomic status according to their geographic origin}

Based on observations made on socioeconomic status in each region of study, we noticed that low-low, low and medium-low status differ according to the development level of each region. We therefore decided to compare seropositivity of children and adolescents belonging to a low-low socioeconomic status for each geographic area studied (Table 3). Subsequently, comparison of seropositivity rates by low-low status for each regions revealed a higher seropositivity for $\operatorname{IgM}$ and protein $\mathrm{A}$ in the UAChR compared with the seropositivity rates found in the Caribbean and the Andes ( $p$-value $<0.05)$. Significant differences in the magnitude of antibody responses of low-low status individuals were also observed across the regions. When compared to Andean and Caribbean 
Table 2 Differences of NDO-LID antibodies according to their socioeconomic status and origin region

\begin{tabular}{|c|c|c|c|c|c|c|c|c|c|c|}
\hline \multirow[t]{4}{*}{ Variable } & \multirow{4}{*}{$\begin{array}{l}\text { Sample size } \\
\text { N (\%) }\end{array}$} & \multirow{2}{*}{\multicolumn{3}{|c|}{$\begin{array}{l}\text { Anti-NDO-LID } \\
\text { protein A }\end{array}$}} & \multicolumn{3}{|c|}{ Anti-NDO-LID IgM } & \multicolumn{3}{|c|}{ Anti- NDO-LID lgG } \\
\hline & & & & & \multirow{3}{*}{$\begin{array}{l}(+) \\
N(\%)\end{array}$} & \multirow{3}{*}{ OR $(95 \% \mathrm{Cl})$} & \multirow{3}{*}{$P$-value } & \multirow[b]{2}{*}{$(+)$} & \multirow{3}{*}{ OR $(95 \% \mathrm{Cl})$} & \multirow{3}{*}{$P$-value } \\
\hline & & $(+)$ & OR $(95 \% \mathrm{Cl})$ & $P$-value & & & & & & \\
\hline & & \multicolumn{3}{|l|}{ N (\%) } & & & & N (\%) & & \\
\hline SEC Low-low & $42(51)$ & $15(36)$ & $10.55(2.2-52.5)$ & 0.001 & $18(43)$ & $6.75(2-22.4)$ & 0.001 & $5(12)$ & $5.3(0.59-47.2)$ & 0.102 \\
\hline $\begin{array}{l}\text { SEC Low or } \\
\text { Medium-low }\end{array}$ & $40(49)$ & \multicolumn{3}{|l|}{$2(5)$} & \multicolumn{2}{|l|}{$4(10)$} & & \multicolumn{2}{|l|}{$1(3)$} & \\
\hline Origin UAChR & $18(22)$ & $12(67)$ & $\begin{array}{l}3.9(1.9-8.1) \\
\text { UAChR vs AR }\end{array}$ & $<0.0001$ & $15(83)$ & $\begin{array}{l}7.5 \text { (1.98-28.3) } \\
\text { UAChR vs AR }\end{array}$ & $<0.0001$ & $3(6)$ & $\begin{array}{l}2.5 \text { (1.6-3.7) } \\
\text { UAChR vs AR }\end{array}$ & 0.136 \\
\hline Origin CR & $43(52)$ & \multicolumn{2}{|l|}{$4(9)$} & & \multicolumn{2}{|l|}{$3(7)$} & & \multicolumn{2}{|l|}{$3(7)$} & \\
\hline Origin AR & $21(26)$ & $1(5)$ & $\begin{array}{l}5.6 \text { (2.5-12.4) } \\
\text { UAChR vs CR }\end{array}$ & & $4(19)$ & $\begin{array}{l}11.9(3.9-36.2) \\
\text { UAChR vs CR }\end{array}$ & & 0 & $\begin{array}{l}1.8(0.7-4.5) \\
\text { UAChR vs CR }\end{array}$ & \\
\hline
\end{tabular}

$O R$ odds ratio, $C$ confidence interval, (+) positive for the presence of anti-NDO-LID antibodies, SEC socioeconomic status, UAChR Uraba-Antioquia-Choco shared region, $C R$ Caribbean region, $A R$ Andean region, $O R$ odds ratio, $C l$ confidence interval, (+) positive for the presence of anti-NDO-LID antibodies

population, significantly higher levels of anti-NDO-LID antibodies were detected in individuals belonging to low-low socioeconomic status that resided in UAChR (Fig. 1c). This data further implies that a higher $M$. leprae frequency of infection occurs in children and adolescents with social inequalities found in a determined geographical region.

\section{Impact of armadillo consumption on serologic responses} Upon questioning, we assessed that 14 individuals reported consumption of armadillo meat at some point (17.1\% of the overall cohort, although for 4 (4.9\%) this data was not documented). We found that anti-NDOLID antibodies could be detected by Protein A in 9 of 14 (64\%) armadillo consumers in contrast to only 8 of 64 (12\%) in those that had not eaten armadillo meat (Table $4, p$-value $<0.0001)$. Furthermore, overall antiNDO-LID antibody levels were higher in children and adolescents that had consumed armadillo meat compared to those that had not ( $p$-value $=0.009$ for Protein A, 0.001 for IgM and 0.013 for IgG) (Fig. 2a). These findings indicate that armadillo consumption is strongly associated with both the rate and level of anti-NDO-LID antibodies.

\section{Impact of BCG re-vaccination status}

A recommendation within leprosy control programs has been the secondary (or initial) BCG vaccination for contacts of diagnosed index cases. Accordingly, we found that $54(66 \%)$ of evaluated children and adolescents had received reinforcement with BCG after their index case had been diagnosed, whereas 16 (19\%) had not received this reinforcement (data was not documented in 12 (15\%) of the study cohort). The frequency of antiNDO-LID antibodies was higher (Table $4, p$-value < $0.01)$, and elevated titers for those detected by Protein A $(p$-value $=0.006), \operatorname{IgM}(p$-value $=0.016)$ and $\operatorname{IgG}(p$ value $=0.007)$, were observed in individuals that had received reinforcement with BCG (Fig. 2b). Thus, a positive BCG immunization status was related to both lower rates and levels of $M$. leprae infection but lack of BCG presented as a risk.

\section{Relationship of serum antibody responses of children with the clinical form of their index case}

It is well documented that contacts of multibacillary (MB) cases are at elevated risk of developing leprosy. We found that the majority $(66 ; 80.5 \%)$ of the study subjects lived with a MB index case while 16 (19.5\%) lived with a paucibacillary (PB) index case. Surprisingly, antiNDO-LID antibodies detected by Protein A, IgM or IgG showed similar rates between $\mathrm{MB}$ or $\mathrm{PB}$ cases ( $p$-value $>0.05$ ). Similarly, differences were not observed in the overall antibody levels between children living with either MB or PB cases ( $p$-value $>0.05$, Fig. 3a). Therefore, in our evaluations the antibody responses of the children did not appear to be influenced by the clinical form of their respective index case.

\section{Relation of antibody responses with the exposure time to the leprosy index case}

Although the frequency of $M$. leprae infection was not impacted to the clinical form of the leprosy index case, we queried if the length of exposure to the leprosy index case could have an impact. Forty-five $(54.9 \%)$ children and adolescents reported an exposure of less than 10 years while 37 (45.1\%) reported exposure periods longer than 10 years (Table 4). When analyzing the presence of circulating antibodies, we found that anti-NDO-LID IgM levels were higher in individuals with $>10$ years of exposure $p$-value $=0.044)($ Fig. $3 \mathrm{~b})$. These data support the hypothesis that prolonged exposure to a leprosy index case increases the risk of $M$. leprae infection. 


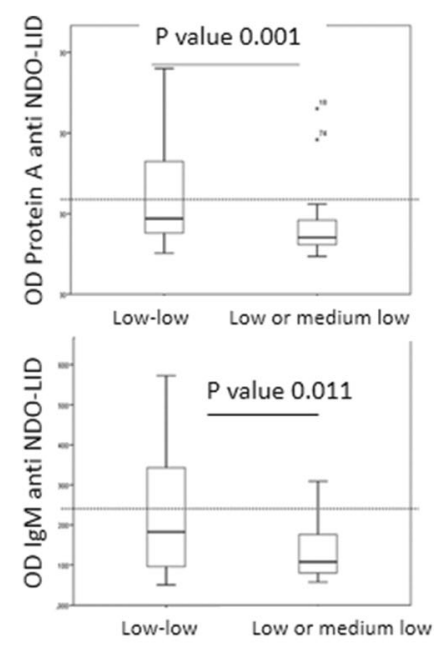

A Socioeconomic status vs Optic density (OD)
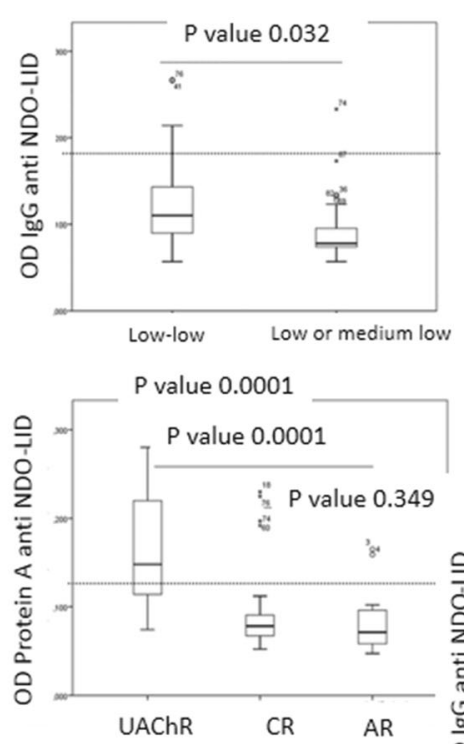

B Geographic region vs Optic density (OD)
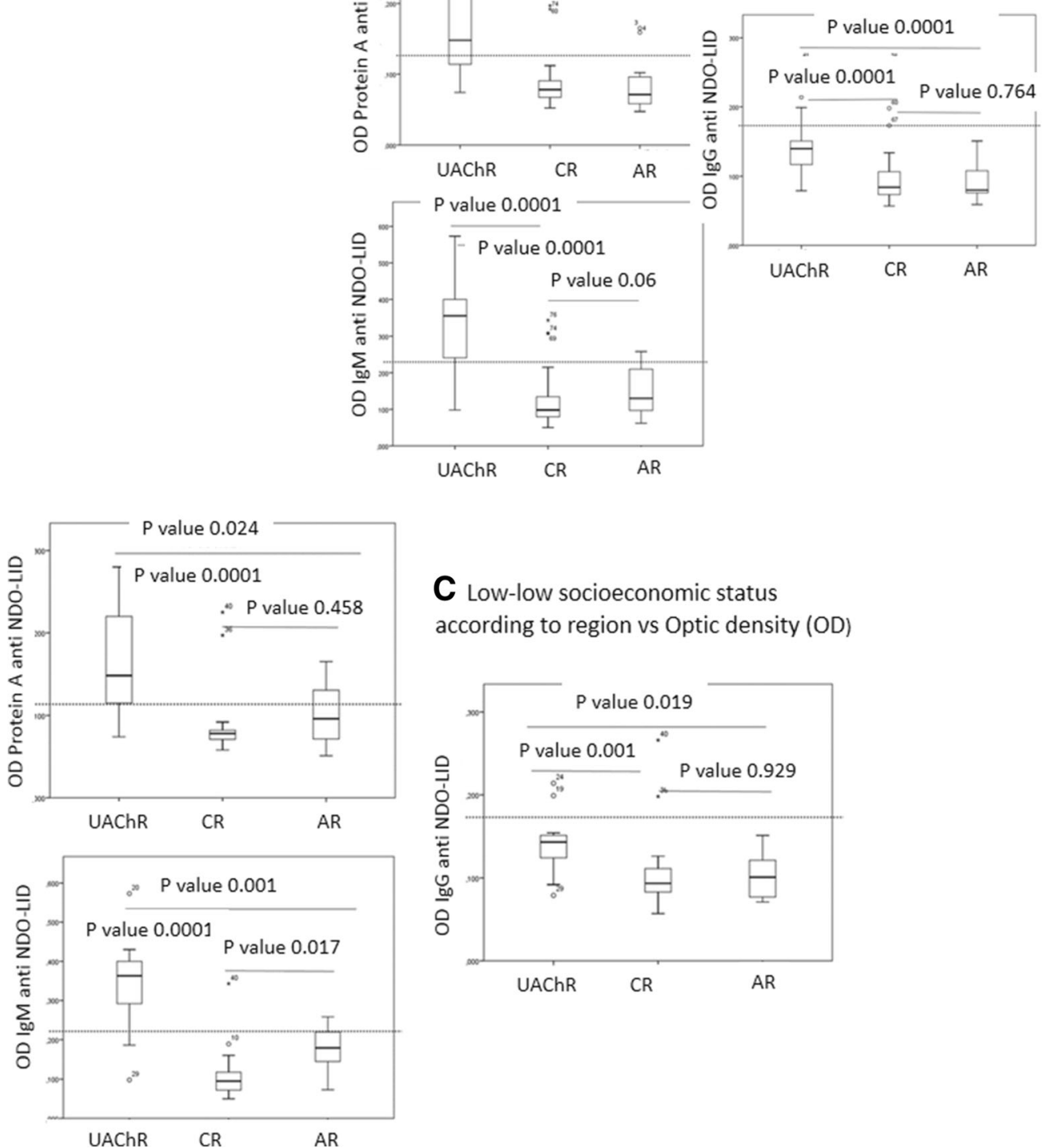

C Low-low socioeconomic status according to region vs Optic density (OD)

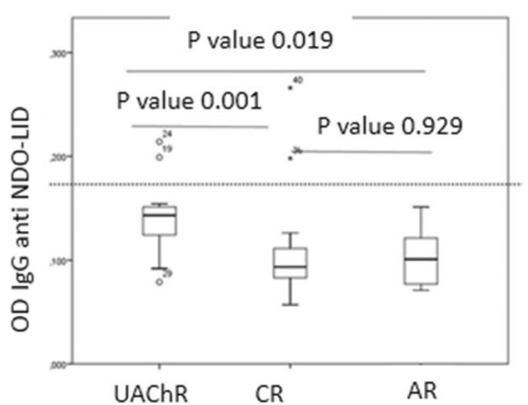

Fig. 1 (See legend on next page.) 
(See figure on previous page.)

Fig. 1 Impact of socioeconomic and geography on M. leprae levels. Anti-natural octyl disaccharide-leprosy IDRI diagnostic (NDO-LID) antibody levels in children and adolescents were measured by ELISA, using either protein A, anti- IgM or anti-lgG to detect responses. In a, samples were stratified by socioeconomic status as either low-low $(n=42)$ or low or medium-low $(n=40)$. In $\mathbf{b}$, samples were stratified by geographic source as UAChR ( $n=18)$, Caribbean Region $(n=43)$, or Andean Region $(n=21)$. In c, samples from the low-low socioeconomic group were subdivided by geographic source as UAChR $(n=41)$, Caribbean Region $(n=43)$, or Andean Region $(n=17)$. Data are displayed as box and whisker plots, with the box representing the Q1 to Q3 interquartile range and the horizontal bar representing the median of the optical density of the samples. Individual dots indicate outliers, and $p$-values are indicated by the lines above each indicated group

Relation of antibody responses with evaluated social and environmental variables

A binomial logistic analysis indicated that the single variable that most strongly associated with the frequency of $M$. leprae infection and increase antibody titers in children and adolescents in Colombia was residency in a geographic region linked to extreme poverty, such as the UAChR shared region $(\mathrm{IgM} / \mathrm{P}$ - value $=0.004$, Protein A/ $P$-value $=0.045)$. Furthermore, it is probable that consumption of armadillo meat, and prolonged exposure to a leprosy case can be related with increase the risk to infection to $M$. leprae (Table 5).

The assessed socio-environmental variables also corresponded with antibody titers when assessed by a multiple lineal analysis, confirming that these variables behave like predictors and do indeed correspond with anti-NDO-LID levels (Protein A $\left(\mathrm{R}=0.59, \mathrm{R}^{2}=0.348, p\right.$ value $=0.0001), \operatorname{IgM}\left(\mathrm{R}=0.746, \mathrm{R}^{2}=0.528, p\right.$-value $=$ $0.0001)$ and $\operatorname{IgG}\left(\mathrm{R}=0.518, \mathrm{R}^{2}=0.268, p\right.$-value $\left.=0.0001\right)$.

The variables related to an increase in antibody titers in children and adolescents in the three endemic regions studied were: 1 . residency in a geographic region linked to extreme poverty, such as the UAChR shared region ( $p$-values $<0.0001$ for IgM and 0.001 for Protein A); $2 .>$ 10 years exposure to a leprosy case ( $p$-values 0.018 for IgM and 0.027 for IgG); and 3. Consumption of armadillo meat ( $p$-value 0.006 for IgM) (Table 6).

These data allowed us to create a series of equations based on the algebraic formula of the multiple lineal regression model, to predict anti-NDO-LID antibody titers (implying $M$. leprae infection) in the children and adolescent populations of the Colombia regions studied. The collinearity of the model was tested using VIF (variance inflation factor), coefficient of determination $R^{2}$, and residual analysis (Additional file 1). The model could be useful to predict IgM anti-NDO-LID antibody titers in the leprosy endemic regions evaluated. The model cannot be validated for Protein A and IgG anti NDO-LID.

\section{Algebraic formula of the multiple regression model}

$$
Y=\beta_{0}+\beta_{1} x_{1}+\beta_{2} x_{2}+\ldots
$$

Y: dependent variable, $x$ : independent variable, $\beta_{0}$ : coefficient (constant) of the dependent variable, $\beta_{1}, \beta_{2}$ : coefficient that signifies a change in the dependent variable when the independent variable is present.

Predictive equation of anti-NDO-LID IgM titers = $0.495 \mathrm{OD}+[-1.42 \mathrm{OD} \times 1$ (not residing in a vulnerable geographic area) or $\times 0$ (residing in a vulnerable geographic area $)]+[0.044 \mathrm{OD} \times 1$ (> 10 years exposure $)$ or $\times 0(<10$ years exposure $)]+[-0.064 \mathrm{OD} \times 1$ (not consuming armadillo meat) or $\times 0$ (consuming armadillo meat)].

\section{Discussion}

Leprosy remains a public health problem in many areas. Establishing the relationship between $M$. leprae infection and socio-environmental factors may help identify why there is greater frequency of infection among juvenile $\mathrm{HHC}$ of leprosy patients relative to other contacts. Considering that leprosy has strong links to poverty $[12,13]$, we examined the rates and levels of serum suggestive of $M$. leprae infection against various indicators of reduced social status in Colombia. In agreement with the general findings of other, we determined that the lower the socioeconomic status of the household inhabited by children and adolescents, the higher rates and levels of

Table 3 Differences of NDO-LID antibodies according to low- low socioeconomic status and geographic area

\begin{tabular}{|c|c|c|c|c|c|c|c|c|c|c|}
\hline \multirow{2}{*}{$\begin{array}{l}\text { Low-low } \\
\text { socioeconomic status } \\
\text { according to the } \\
\text { geographic area }\end{array}$} & \multirow{2}{*}{$\begin{array}{l}\text { Sample } \\
\text { size } \\
\text { N (\%) }\end{array}$} & \multicolumn{3}{|c|}{ Anti-NDO-LID protein A } & \multicolumn{3}{|c|}{ Anti-NDO-LID IgM } & \multicolumn{3}{|c|}{ Anti- NDO-LID IgG } \\
\hline & & $\begin{array}{l}+(+) \\
N(\%)\end{array}$ & OR $(95 \% \mathrm{Cl})$ & $P$-value & $\begin{array}{l}(+) \\
N(\%)\end{array}$ & OR $(95 \% \mathrm{Cl})$ & $P$-value & $\begin{array}{l}(+) \\
N(\%)\end{array}$ & OR $(95 \% \mathrm{Cl})$ & $P$-value \\
\hline Low-low- UAChR & $17(41)$ & $12(71)$ & \multirow{2}{*}{$\begin{array}{l}3.6(1.6-7.9) \\
\text { UAChR vs CR }\end{array}$} & \multirow[t]{2}{*}{0.001} & $15(88)$ & \multirow{2}{*}{$\begin{array}{l}8.9(2.4-33.2) \\
\text { UAChR vs CR }\end{array}$} & \multirow[t]{2}{*}{$<0.0001$} & $3(18)$ & \multirow{2}{*}{$\begin{array}{l}2.5(1.6-3.7) \\
\text { UAChR vs CR }\end{array}$} & \multirow[t]{2}{*}{0.474} \\
\hline Low-low - CR & $18(43)$ & $2(11)$ & & & $1(6)$ & & & $2(11)$ & & \\
\hline Low-low- AR & $7(17)$ & $1(14)$ & $\begin{array}{l}2(1.04-3.9) \\
\text { UAChR vs AR }\end{array}$ & & $2(29)$ & $\begin{array}{l}3.1(1-11) \\
\text { UAChR vs AR }\end{array}$ & & 0 & $\begin{array}{l}1.5(1.2-2) \\
\text { UAChR vs AR }\end{array}$ & \\
\hline
\end{tabular}

UAChR Uraba-Antioquia-Choco shared region, $C R$ Caribbean region, $A R$ Andean region, $O R$ odds ratio, $C l$ confidence interval, (+) positive for the presence of anti-NDO-LID antibodies 
Table 4 Differences of NDO-LID antibodies according to armadillo meat intake, BCG re-vaccination, and time of exposure

\begin{tabular}{|c|c|c|c|c|c|c|c|c|c|c|}
\hline \multirow[t]{3}{*}{ Variable } & \multirow[t]{3}{*}{ N (\%) } & \multicolumn{3}{|c|}{ Anti-NDO-LID protein A } & \multicolumn{3}{|c|}{ Anti-NDO-LID IgM } & \multicolumn{3}{|c|}{ Anti- NDO-LID lgG } \\
\hline & & $\overline{(+)}$ & OR $(95 \% \mathrm{Cl})$ & $P$-value & $\overline{(+)}$ & OR $(95 \% \mathrm{Cl})$ & $P$-value & $\overline{(+)}$ & OR $(95 \% \mathrm{Cl})$ & $P$-value \\
\hline & & \multicolumn{3}{|l|}{ N (\%) } & \multicolumn{3}{|l|}{$N(\%)$} & \multicolumn{3}{|l|}{ N (\%) } \\
\hline Armadillo meat intake & $14(17)$ & $9(64)$ & $12.6(3.3-47)$ & $<0.0001$ & $11(79)$ & $17.6(4.2-74)$ & $<0.0001$ & $3(21)$ & $5.5(0.9-31)$ & 0.79 \\
\hline Non armadillo meat intake & $64(78)$ & $8(12)$ & & & $11(17)$ & & & $3(5)$ & & \\
\hline BCG re-vaccination & $54(66)$ & $9(17)$ & $0.2(0.059-0.67)$ & 0.002 & $12(22)$ & $0.22(-0.07-0.72)$ & 0.008 & $3(6)$ & $0.25(0.046-1.4)$ & 0.118 \\
\hline No BCG re-vaccination & $16(19)$ & $8(50)$ & & & $9(56)$ & & & $3(19)$ & & \\
\hline Time of exposure $<10$ years & $45(55)$ & $6(13)$ & $1.6(1.02-2.56)$ & 0.068 & $8(18)$ & $1.6(1.05-2.6)$ & 0.041 & $1(2)$ & $1.9(1.2-3)$ & 0.086 \\
\hline Time of exposure $>10$ years & $37(45)$ & $11(30)$ & & & $14(38)$ & & & $5(14)$ & & \\
\hline
\end{tabular}

OR odds ratio, $\mathrm{Cl}$ confidence interval, (+) positive for the presence of anti-NDO-LID antibodies

antibodies against the diagnostic conjugate NDO-LID. Residency in a geographic region linked to extreme poverty and prolonged ( $>10$ years) exposure to a leprosy case were very influential, as was reported consumption of armadillo meat. Although limited to convenience sampling and a small sample collection that does not represent all Colombian regions, at a time when leprosy control program activities are generally being scaled back or integrated into general health systems, our data and methods could help focus control efforts, inform education campaigns and enhance the overall output of leprosy-specific programs.

It is widely reported and accepted that $\mathrm{HHC}$ of $\mathrm{MB}$ patients have a higher risk of $M$. leprae infection and subsequent development of leprosy than $\mathrm{HHC}$ of PB patients [14]. In contrast with the reported data of Amorim et al. [15], in which elevated antibody responses were found in $\mathrm{HHC}$ of $\mathrm{MB}$ patients compared to $\mathrm{HHC}$ of $\mathrm{PB}$ patients, our analyses found no significant differences in either anti-NDO-LID frequencies or levels when juvenile contacts were stratified by index case presentation. Although our previous data did not statistically relate serum antibody responses with duration of exposure to a patient [6] others have linked this with risk of M. leprae infection [15] and we therefore decided to assess this environmental variable and relationship among subgroups in the current study. Accordingly, we observed that individuals with $>10$ years exposure had significantly greater rates of seropositivity than those with < 10 years exposure. These results suggest that those with a longer length of residency with a leprosy index case, and therefore greater cumulative exposure, have a greater likelihood of infection and of developing the disease.

Knowing that leprosy is associated with poverty is not particularly beneficial in focusing control efforts in

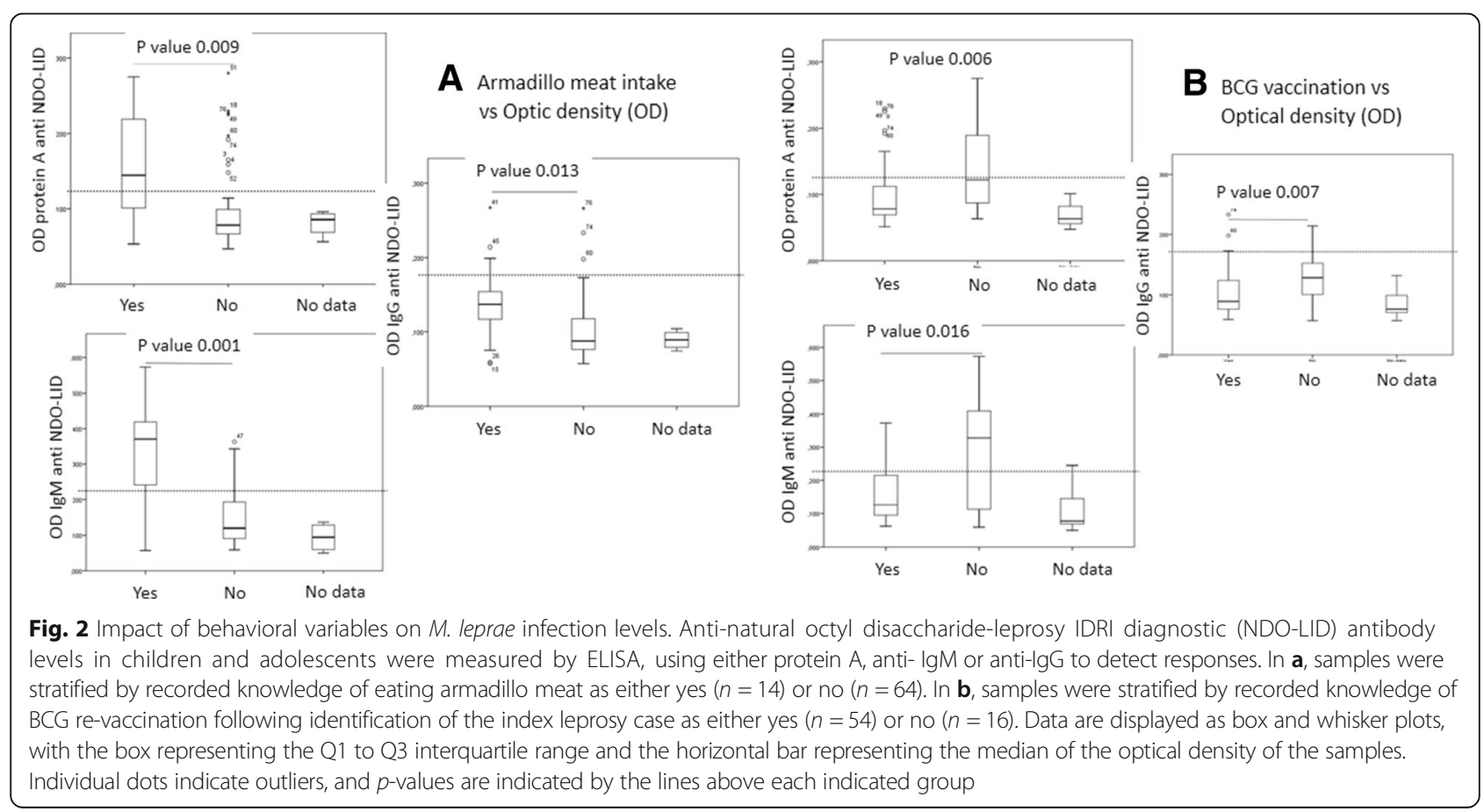




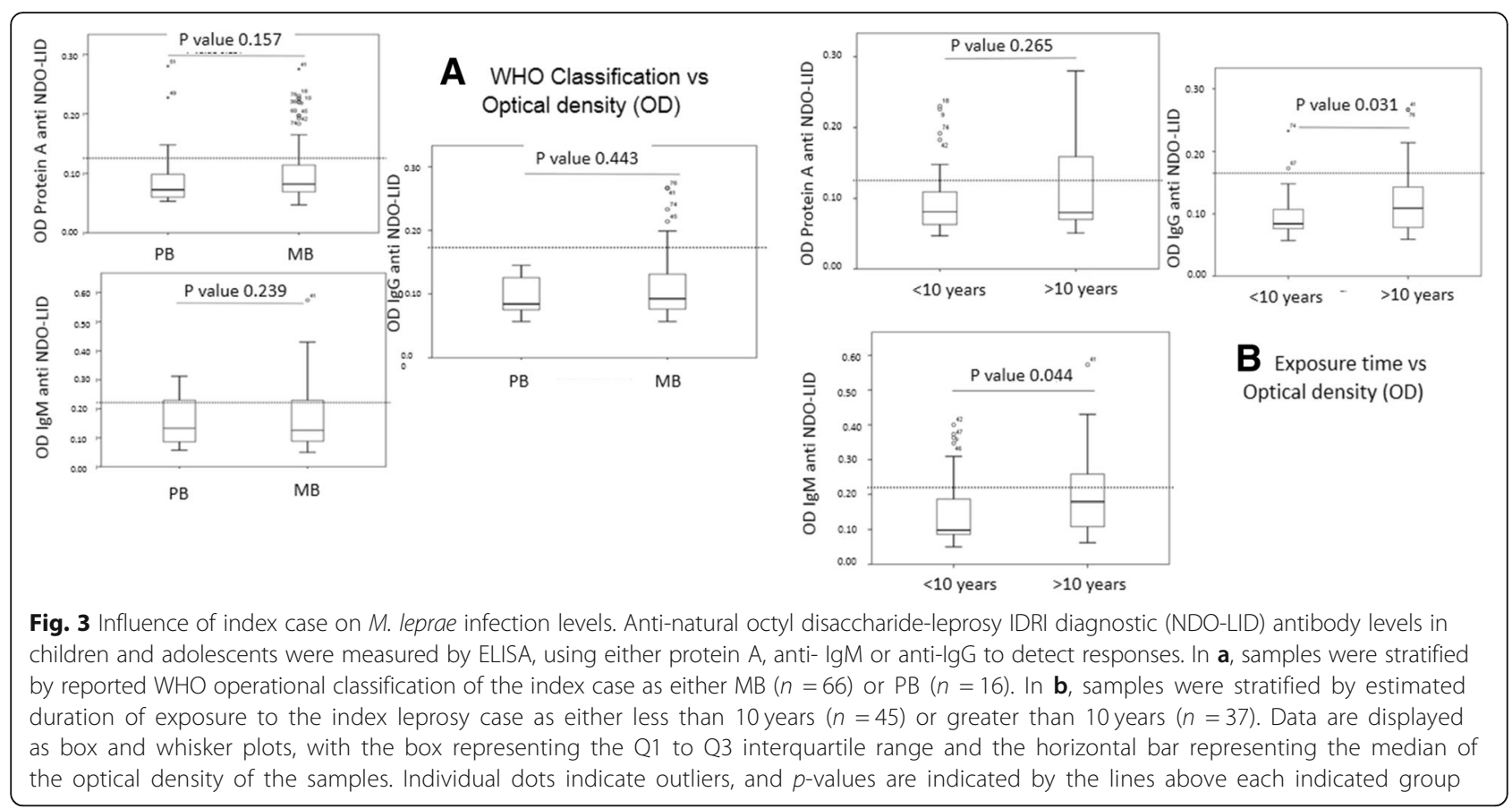

Colombia because it has the third lowest distribution of wealth of any country in the world [16]. Our study indicates that the geographic region in which children and adolescents reside is, however, an important variable that presents risk for $M$. leprae infection. Serologic data from three distinct geographic regions (Uraba-AntioquiaChoco shared region, the Caribbean region, and the Andean region), revealed that juveniles in the UAChR region had a higher $M$. leprae infection rates than the other regions. These results can be explained in part by the inherent conditions of this region. UAChR is characterized for its poor public health conditions, being one of the most unequal distribution of wealth regions in Colombia, and for having parts of its territory lacking in fundamental public services like basic sanitation as well as electricity and water supply. This finding agrees with observations made in 139 municipalities in Tocantins, Brazil [13], where municipalities with a higher vulnerability and social inequality presented with increases in leprosy onset and spread of $M$. leprae.
When evaluating the relative impacts of either region of residence or socioeconomic status on $M$. leprae infection in the study populations, we found that, rather than belonging to a low-low socioeconomic status, inhabiting a vulnerable geographic region (without sanitary and public services like UAChR) was the key social variable that resulted in increased frequency of $M$. leprae infection. This was heightened by the fact that these vulnerable geographic regions have unfavorable socioeconomic conditions such as malnutrition, low schooling levels and absence of leprosy control program interventions $[12,17]$.

It is noteworthy to the region that $M$. leprae infection has been associated with armadillo hunting and manipulation [18, 19]. Given that leprosy can be considered somewhat zoonotic, that $M$. leprae-infected armadillos have been found in Colombia, and that armadillos are often used as a meat source, in traditional medicine and as pets, we hypothesized that armadillo consumption could be of great importance in the studied population

Table 5 Differences NDO-LID seropositivity according to social and environmental factors evaluated

\begin{tabular}{|c|c|c|c|c|c|c|c|c|c|}
\hline Factors & $\begin{array}{l}\text { IgM Anti-NDO-LID } \\
\text { (ExpB) }\end{array}$ & $95 \% \mathrm{Cl}$ & $P$-value & $\begin{array}{l}\text { Protein A Anti } \\
\text { NDO-LID (ExpB) }\end{array}$ & $95 \% \mathrm{Cl}$ & $P$-value & $\begin{array}{l}\lg G \text { Anti NDO-LID } \\
\text { (ExpB) }\end{array}$ & $95 \% \mathrm{Cl}$ & $P$-value \\
\hline Geographic area & 19.8 & $2.6-150$ & 0.004 & 6.5 & $1.04-41$ & 0.045 & 0.678 & $0.3-13$ & 0.8 \\
\hline Time of exposure & 0.186 & $0.03-1.1$ & 0.063 & 0.32 & $0.068-1.55$ & 0.16 & 0.18 & $0.02-1.8$ & 0.15 \\
\hline Armadillo meat intake & 9.4 & $0.97-90$ & 0.053 & 8.1 & $0.92-70$ & 0.059 & 7.4 & $0.32-170$ & 0.21 \\
\hline BCG re-vaccination & 1.07 & $0.27-4.2$ & 0.92 & 1.77 & $0.34-9.1$ & 0.496 & 1.5 & $0.18-12$ & 0.719 \\
\hline Socioeconomic status & 1.87 & $0.35-9.8$ & 0.462 & 3.3 & $0.48-22$ & 0.22 & 3.9 & $0.26-58$ & 0.32 \\
\hline
\end{tabular}

ExpB Multivariate-adjusted Odds ratio, $\mathrm{Cl}$ confidence interval 
Table 6 Differences NDO-LID antibodies according to social and environmental factors evaluated

\begin{tabular}{|c|c|c|c|c|c|c|}
\hline \multirow[t]{2}{*}{ Factors } & \multicolumn{2}{|c|}{ IgM Anti-NDO-LID } & \multicolumn{2}{|c|}{$\begin{array}{l}\text { Protein A } \\
\text { Anti-NDO-LID }\end{array}$} & \multicolumn{2}{|c|}{ IgG Anti- NDO-LID } \\
\hline & $\overline{O D^{a}}$ & $P$-value & $\overline{\mathrm{OD}^{a}}$ & $P$-value & $\overline{O D^{a}}$ & $P$-value \\
\hline $\begin{array}{l}\text { Socioeconomic status - Geographic area - } \\
\text { Armadillo meat intake- BCG re-vaccination- } \\
\text { time of exposure }\end{array}$ & $0.495^{a}$ & $<0.0001$ & $0.270^{a}$ & $<0.0001$ & $0.169^{a}$ & $<0.0001$ \\
\hline Geographic area & $-1.42^{b}$ & $<0.0001$ & $-0.053^{b}$ & 0.001 & $-0.022^{b}$ & 0.115 \\
\hline Time of exposure & $0.044^{b}$ & 0.018 & - & - & $0.021^{\mathrm{b}}$ & 0.027 \\
\hline Armadillo meat intake & $-0.064^{\mathrm{b}}$ & 0.006 & $-0.02^{b}$ & 0.136 & $-0.016^{\mathrm{b}}$ & 0.152 \\
\hline BCG re-vaccination & $0.001^{b}$ & 0.940 & $-0.006^{\mathrm{b}}$ & 0.384 & $0.003^{b}$ & 0.648 \\
\hline Socioeconomic status & $-0.009^{b}$ & 0.651 & $-0.017^{b}$ & 0.160 & -0.018 & 0.079 \\
\hline
\end{tabular}

${ }^{\mathrm{a} O D}$ : optic density average, ${ }^{\mathrm{b}}$ change of OD anti-NDO-LID

$[9,20]$. While Schmitt et al. [21] could not relate armadillo meat consumption with $M$. leprae infection in Brazil, arguing not only that cooking the meat would kill the mycobacterium but also that there is a lack of evidence for gastrointestinal transmission, we found that armadillo meat consumption correlated with higher frequencies of $M$. leprae infection in children and adolescents. This is consistent with other recent findings that armadillo meat consumption is related to a higher proportion of leprosy cases $[19,20]$.

Among the factors that appeared protective, BCG immunization was associated with reduced $M$. leprae infection frequencies among the children and adolescents in our study cohorts. The BCG vaccine can enhance cellular immune responses against $M$. leprae, and immunization with BCG has been recommended for HHC of diagnosed leprosy index cases [22]. Our findings are therefore compatible with both our previous research as well studies in Brazilian populations. As a preemptive approach to reduce the emergence of new cases and enable $M$. leprae elimination, it has recently been suggested that seropositive individuals that have asymptomatic or sub-clinical $M$. leprae infection receive BCG re-vaccination as well as treatment with rifampicin to serve as an early intervention to alter the natural course of infection [23, 24]. Our data provide further evidence for the implementation of prophylactic measures within high risk juvenile and other HHC populations.

In summary, our assessment among Colombian children and adolescents in contact with leprosy cases reveals that risk of $M$. leprae infection is increased by: residency in a vulnerable geographic region; > 10 years exposure to the leprosy index case; and consumption of armadillo meat. These results are similar to those obtained in other socioeconomic analyses [12, 13, 18] strengthening our understanding of the impact of environmental and behavioral variables on the risk of becoming infected with $M$. leprae and potentially developing leprosy. We propose that regular monitoring of the serum anti-NDO-LID antibodies in children and adolescents, and the construction of predictive models for anti-M. leprae antibody titers, would be useful among HHC to aid both the early diagnosis of new leprosy cases and track transmission of $M$. leprae. Together, this information can be used to focus control efforts.

\section{Conclusions}

Despite being classified by the WHO as a disease in elimination phase, our results show that, in accordance with the national control program statistics [25], leprosy continues to be a public health issue in several regions in Colombia. This situation points out either failures or a lack of implementation of the eradication strategies proposed by the WHO.

This study has also proven that active $M$. leprae transmission persists in child and adolescent population, mainly in those populations located in vulnerable geographic regions with little presence from government, with armadillo meat consumption traditions, and that are subject to a long exposure to leprosy cases. Therefore, these findings show us that as long as the government does not intervene on the critical socioenvironmental variables distressing juvenile population, leprosy elimination will only be a utopia achieved on the desk of the national control program.

\section{Additional file}

Additional file 1: Validation of multiple regression approach for lgM anti NDO-LID. (DOCX 5547 kb)

Abbreviations

AR: Andean Region; BCG: Bacillus Calmette-Guerin; CR: Caribbean Region; HHC: Household contact; ICMT: Instituto Colombiano de Medicina Tropical: M. leprae: Mycobacterium leprae; MB: Multibacillary; NDO-LID: Anti- Natural Octyl Disaccharide-Leprosy IDRI Diagnostic; PB: Paucibacillary; PGL-I: Phenolic glycolipid-I; UAChR: Uraba-Antioquia-Choco Region 


\section{Acknowledgements}

The authors would like to thank the children and their families who voluntarily participated in this study, as well as the health personnel of each region studied. Also, the authors want to thank Simon RobledoCardona for Spanish-English translation.

\section{Authors' contributions}

The study was designed by NCC. The Children and adolescents were evaluated by HS, HRM, and JCB. The serologic evaluation was performed by JCB and HRM using antigen provided by MSD. The data analysis was performed by HS and NCC. The manuscript was written by HS and NCC. MSD made critical revisions. All authors have read and approved the manuscript.

\section{Funding}

Fondation Mérieux, Lyon, France funded by a grant to Nora Cardona-Castro to design of the study, data collection, analysis, and writing. Héctor Serrano-Coll had financial support from Colciencias (grant 727-2015) to perform analysis and writing. The funding bodies had no role in the design of the study, analysis, interpretation of data, and in writing the manuscript.

\section{Availability of data and materials}

The datasets generated and/or analyzed during the current study are not publicly available but are available from the corresponding author on reasonable request.

\section{Ethics approval and consent to participate}

This study was approved by the Instituto Colombiano de Medicina Tropical Universidad CES ethics committee. After the aims of the study were explained, an informed consent form was signed by the guardians of participating children

\section{Consent for publication}

Not applicable.

\section{Competing interests}

Malcolm Duthie provides antigen to commercial companies for fabrication of rapid diagnostic tests. The other authors declare having no conflict of interests. Nora Cardona-Castro is a member of the editorial board (Associate Editor) of BMC Infectious Diseases.

\section{Author details}

${ }^{1}$ Graduate School - Universidad CES, Medellín, Colombia. Instituto Colombiano de Medicina Tropical-Universidad CES, Sabaneta, Colombia. ${ }^{3}$ Infectious Disease Research Institute, Seattle, USA. ${ }^{4}$ Instituto Colombiano de Medicina Tropical, Faculty of Medicine, Escuela de Graduados - Universidad CES, Cra 43 A \# 52 Sur 99., Sabaneta, Colombia.

\section{Received: 10 October 2018 Accepted: 22 May 2019}

\section{Published online: 13 June 2019}

\section{References}

1. Serrano-Coll H, Acevedo-Saenz L, Cardona-Castro N. A hypothetical role for notch signaling pathway in immunopathogenesis of leprosy. Med Hypotheses. 2017;109:162-9.

2. Nsagha DS, Bamgboye EA, Assob JCN, Njunda AL, Kamga HLF, Zoung-Kanyi Bissek A-C, et al. Elimination of leprosy as a public health problem by 2000 AD: an epidemiological perspective. Pan Afr Med J. 2011;9:4.

3. Barreto JG, Frade MAC, Bernardes Filho F, da Silva MB, Spencer JS, Salgado CG. Leprosy in children. Curr Infect Dis Rep. 2017;19(6):23.

4. Romero-Montoya IM, Beltrán-Alzate JC, Ortiz-Marín DC, Diaz-Diaz A, Cardona-Castro N. Leprosy in Colombian children and adolescents. Pediatr Infect Dis J. 2014;33(3):321-2.

5. Cardona-Castro N. Leprosy in Colombia. Curr Trop Med Rep. 2018;5:85 https://doi.org/10.1007/s40475-018-0145-7.

6. Romero-Montoya M, Beltran-Alzate JC, Cardona-Castro N. Evaluation and Monitoring of Mycobacterium leprae Transmission in Household Contacts of Patients with Hansen's Disease in Colombia. PLoS Negl Trop Dis. 2017; 11(1):e0005325. https://doi.org/10.1371/journal.pntd.0005325.

7. Muñoz M, Beltrán-Alzate JC, Duthie MS, Serrano-Coll H, Cardona-Castro N. Comparison of enzyme-linked immunosorbent assay using either natural Octyl disaccharide-leprosy IDRI diagnostic or phenolic glycolipid-I antigens for the detection of leprosy patients in Colombia. Am J Trop Med Hyg. 2018;98(1):274-7.

8. Mistry N, Kuruwa S, Pandya S, Minda R, Shetty V. Childhood Leprosy Revisited. Ped Oncall. 2016:13(4):83-92.

9. Cardona-Castro N, Beltrán JC, Ortiz-Bernal A, Vissa V. Detection of Mycobacterium leprae DNA in nine-banded armadillos (Dasypus novemcinctus) from the Andean region of Colombia. Lepr Rev. 2009; 80(4):424-31.

10. Serrano-Coll H, Muñoz M, Camilo Beltrán J, Duthie MS, Cardona-Castro N. Anti-natural octyl disaccharide-leprosy IDRI diagnostic (NDO-LID) antibodies as indicators of leprosy reactions and neuritis. Trans R Soc Trop Med Hyg. 2017;111(3):125-31.

11. Departamento Administrativo Nacional de Estadística. Estratificación socioeconómica. Available: https://www.dane.gov.co/files/geoestadistica/ Preguntas_frecuentes_estratificacion.pdf. Accesed 23 Feb 2019 and 27 May 2019.

12. Simionato de Assis I, Arcoverde MAM, Ramos ACV, Alves LS, Berra TZ, Arroyo $\mathrm{LH}$, et al. Social determinants, their relationship with leprosy risk and temporal trends in a tri-border region in Latin America. PLoS Negl Trop Dis. 2018;12(4):e0006407.

13. Monteiro LD, Mota RMS, Martins-Melo FR, Alencar CH, Heukelbach J. Social determinants of leprosy in a hyperendemic state in North Brazil. Rev Saude Publica. 2017:51:70.

14. Cabral-Miranda W, Chiaravalloti Neto F, Barrozo LV. Socio-economic and environmental effects influencing the development of leprosy in Bahia, North-Eastern Brazil. Trop Med Int Health TM IH. 2014:19(12):1504-14.

15. Amorim FM, Nobre ML, Ferreira LC, Nascimento LS, Miranda AM, Monteiro GRG, et al. Identifying leprosy and those at risk of developing leprosy by detection of antibodies against LID-1 and LID-NDO. PLoS Negl Trop Dis. 2016;10(9):e0004934.

16. Oxford Business Group. New programmes in Colombia to decrease inequality. 2016. Available in: https://oxfordbusinessgroup.com/analysis/ growing-together-raft-new-programmes-are-aimed-decreasing-inequality. Accesed 30 Jan 2019 and 27 May 2019

17. Pedrosa VL, Dias LC, Galban E, Leturiondo A, Palheta J, Jr, Santos M, et al. Leprosy among schoolchildren in the Amazon region: A cross-sectional study of active search and possible source of infection by contact tracing. PLoS Negl Trop Dis. 2018;12(2):e0006261. https://doi.org/10.1371/journal. pntd.0006261.

18. Clark BM, Murray CK, Horvath LL, Deye GA, Rasnake MS, Longfield RN. Casecontrol study of armadillo contact and Hansen's disease. Am J Trop Med Hyg. 2008;78(6):962-7.

19. Domozych R, Kim E, Hart S, Greenwald J. Increasing incidence of leprosy and transmission from armadillos in Central Florida: a case series. JAAD Case Rep. 2016;2(3):189-92.

20. Truman RW, Singh P, Sharma R, Busso P, Rougemont J, Paniz-Mondolfi A, et al. Probable zoonotic leprosy in the southern United States. N Engl J Med. 2011;364(17):1626-33.

21. Schmitt JV, Dechandt IT, Dopke G, Ribas ML, Cerci FB, Viesi JMZ, et al. Armadillo meat intake was not associated with leprosy in a case contro study, Curitiba (Brazil). Mem Inst Oswaldo Cruz. 2010;105(7):857-62.

22. Zenha EMR, Wambier CG, Novelino AL, de Andrade TAM, Ferreira MAN, Frade MAC, et al. Clinical and immunological evaluation after BCG-id vaccine in leprosy patients in a 5-year follow-up study. J Inflamm Res. 2012; 5:125-35.

23. Düppre NC, Camacho LAB, Sales AM, Illarramendi X, Nery JAC, Sampaio EP, et al. Impact of $P G L-I$ seropositivity on the protective effect of BCG vaccination among leprosy contacts: a cohort study. PLoS Negl Trop Dis. 2012;6(6):e1711.

24. Dos Santos DS, Duppre NC, Sarno EN, Pinheiro RO, Sales AM, Nery JADC, et al. Chemoprophylaxis of leprosy with rifampicin in contacts of multibacillary patients: study protocol for a randomized controlled trial. Trials. 2018;19(1):244.

25. Ministerio de Salud y Protección social Colombia. Guía de atención integral de la lepra. 2012. Available: Guías de promoción de la salud y prevención de enfermedades en la salud pública. https://www.minsalud.gov.co/ Documentos\%20y\%20Publicaciones/ GUIA\%20DE\%20ATENCI\%C3\%93N\%20DE\%20LEPRA.pdf. Accesed 27 May 2019.

\section{Publisher's Note}

Springer Nature remains neutral with regard to jurisdictional claims in published maps and institutional affiliations. 